B.-O. Dozo, «Le héros dans La Bataille littéraire après la Grande Guere: une représentation au service d'une littérature nationalebelge», dans Y.HAMELetM.BOUCHARD(dir),Portraitdel'hommedelettres en héros, @nalyses,hiver2006

\title{
Björn-Olav Dozo
}

\section{Le héros dans La Bataille littéraire après la Grande Guerre : une représentation au service d'une littérature nationale belge}

La Belgique, au sortir de la Première Guerre mondiale, est un petit pays qui vient de se faire une place dans la cour des grands. Ses souffrances durant la guerre, sa résistance dans les tranchées de l'Yser, les preuves de son courage assoient à l'extérieur de ses frontières un discours de reconnaissance et d'admiration. Ce discours lui vaudra d'être reconnue comme une puissance politique avec laquelle il faut compter. La Belgique n'est plus seulement la petite nation industrieuse, née un peu par hasard des aléas de l'histoire pour servir de tampon entre les grands pays de la première moitié du XIX ${ }^{\mathrm{e}}$ siècle.

Elle était devenue une puissance coloniale en 1908, en acceptant de reprendre l'État indépendant du Congo, qui appartenait jusque-là en propre au roi Léopold II. À la sortie de la Première Guerre, le mandat des colonies allemandes du Ruanda-Urundi lui est confié par la Société des Nations lors du traité de Versailles du 28 juin 1919. Ce mandat, et donc cette présence forte dans les colonies, soulignent le rôle important de la Belgique sur la scène internationale à cette époque.

Cette reconnaissance internationale servira d'argument dans le champ littéraire, notamment lors de la création de l'Académie royale de langue et littérature française de Belgique. Ainsi, Jules Destrée, alors ministre des Arts et des Sciences, insiste sur le rôle de la Belgique lors de la guerre dans son rapport au Roi, et sur l'opportunité pour elle de jouer le rôle d'un lieu de regroupement international francophone :

L'Académie pourra appeler à elle un nombre limité d'écrivains ou de philologues de nationalité étrangère choisis non seulement en France, mais aussi au Canada, en Suisse romande, en Italie, en Roumanie, en Tchéco-Slovaquie, dans tous les pays 
B.-O. Dozo, «Le héros dans La Bataille littéraire après la Grande Guere: une représentation au service d'une littérature nationalebelge», dans Y.HAMELetM.BOUCHARD(dir),Portraitdel'hommedelettres en hérns, @nalyses, hiver2006

où le français est parlé, honoré, cultivé, et qui sont comme les provinces intellectuelles de la civilisation française. Aucun lien ne rattache les uns aux autres, à l'heure actuelle, ces divers centres de culture; il a paru que la Belgique, tant par sa situation géographique qu'à raison du prestige que lui ont valu les épreuves de la guerre, était spécialement qualifiée pour essayer de réaliser un groupement international de cette espèce. (1922, p. 7)

Cette promotion internationale de la « grandeur » d'une Belgique unie n'est pourtant qu'un leurre : en interne, les tensions communautaires se font sentir fortement, et le même Destrée, en 1912 déjà, écrivait sa fameuse Lettre au Roi sur la séparation de la Wallonie et de la Flandre ("Sire, il n'y a pas de Belges. Il n'y a que des Flamands et des Wallons »). Celle-ci met en évidence l'existence de deux communautés bien distinctes, tant linguistiquement que socialement et culturellement. La guerre n'aura finalement été qu'une parenthèse dans un processus de longue haleine. Ce processus de scission reprendra de plus belle en 1923, avec la flamandisation de l'Université de Gand, qui suscitera de nombreuses réactions, en Belgique comme dans la presse étrangère. Pour rester avec le même exemple, il faut souligner la publication par Destrée chez Plon, en 1923, d'un ouvrage intitulé $W$ allons et Flamands, sorte de retour à ses premières préoccupations.

Notre objectif n'est pas de revenir sur le fondement ontologique ou idéologique de ce processus de séparation. Nous plaçons seulement les cadres nécessaires à l'analyse de l'utilisation de l'image du héros par une revue littéraire, La Bataille littéraire, au sortir de la Première Guerre. Il faut d'ailleurs insister sur le fait que cette utilisation est propre à la revue, et ne constitue en aucune manière une norme pour l'époque en Belgique.

\section{La Bataille littéraire}

Le premier numéro de La Bataille littéraire parait le 23 janvier 1919. Hebdomadaire, la revue se présente sous la forme d'un journal d'un, puis de deux feuillets. Elle appartient à «la quarantaine de revues 
B.-O. Dozo, «Le héros dans La Bataille littéraire après la Grande Guere: une représentation au service d'une littérature nationalebelge», dans Y.HAMELetM.BOUCHARD(dir),Portraitdel'hommedelettres en héros, @nalyses,hiver2006

spécifiquement littéraires qui paraissent entre 1919 et 1923 [et qui] illustrent le renouveau des lettres après la Grande Guerre »(1998, p. 31). Aucune étude n'a été réalisée à son sujet: elle ne rencontre aucun courant avant-gardiste, comme Résurrection, Ça ira! ou Lumière. Au contraire, La Bataille littéraire, comme on se propose de le montrer, se situe assez loin des enjeux esthétiques propres à l'époque. Pour analyser sa position, il nous faut en livrer une lecture problématisée, permettant de situer ses orientations littéraires et politiques.

L'éditorial du premier numéro, intitulé «Premiers coups de canon », fixe les objectifs de la revue.

Ohé! donc debout ceux qui s'engagent à nous aider! La Belgique a cessé d'être un petit pays. En même temps qu'on travaille à sa reconstruction matérielle, il faut que jaillisse de ses ruines une efflorescence intellectuelle et morale digne des sacrifices que le Peuple a supportés. [...] C'est par ses Arts, et sa Littérature surtout, qu'une Nation s'assure dans le Monde une grandeur souveraine. (1919a, p. 1)

Ces quatre lignes permettent de situer les conceptions de l'énonciateur: au sortir de la guerre, les littérateurs qui le souhaitent doivent «s'engager», comme on s'enrôle dans l'armée, pour aider à reconstruire intellectuellement la nation. La littérature est conçue comme un moyen de servir la patrie, une façon d'être utile au peuple qui s'est sacrifié. C'est la cause de la Belgique qu'il faut défendre. Et derrière ce discours qui soutient la reconstruction de la Belgique se dessine une certaine conception de la littérature :

À des temps nouveaux, il faut des lettres nouvelles.

La radieuse flambée de la Jeune Belgique n'est plus qu'un souvenir! Depuis quatre vingt-quinze, une littérature de chefs de bureau s'est appliquée à l'éteindre sous les arrosoirs de la banalité. Il y a pourtant toujours chez nous d'excellents poètes et de grands prosateurs; ce qui nous manque, c'est un véritable mouvement littéraire [...]. Par le dessin et la critique, nous illustrerons une série de biographies «d'hommes du jour», que 
B.-O. Dozo, «Le héros dans La Bataille littéraire après la Grande Guere: une représentation au service d'une littérature nationalebelge», dans Y.HAMELetM.BOUCHARD(dir),Portraitdel'hommedelettres en hérns, @nalyses, hiver2006

nous choisirons parmi les types bien représentatifs de nos diverses activités nationales. (1919a, p. 1)

L'ennemi est pointé du doigt: «la littérature de chefs de bureau », qui a fait dévier les littérateurs belges du chemin ouvert par La Jeune Belgique. Ce discours reproduit un topos qui existait déjà lors de la création de La Jeune Belgique, qui s'opposait quant à elle aux "perruques» de la Thérésienne, la première académie royale.

La Bataille littéraire entend reprendre le flambeau de La Jeune Belgique, contre les « chefs de bureau », en établissant un panthéon nouveau. Ce panthéon, elle l'a annoncé avec sa "série de biographies d'"hommes du jour" » signalée dans l'extrait précédent.

\section{La consécration des auteurs belges}

Pour ce faire, La Bataille littéraire publie à chaque numéro, en première page, en colonne centrale, des biographies d'auteurs particulièrement illustres à ses yeux. Ce sont ces auteurs que nous qualifierons de "héros littéraires ». Le mot héros (ou plus exactement « héroïque ») n'est explicitement utilisé qu'une seule fois dans les biographies, pour évoquer Verhaeren: «Verhaeren! Ô Maître douloureux; poète héroïque et sanglant! » (19191, p. 1). Son statut particulier est d'ailleurs directement évoqué dans la première phrase du premier portrait biographique. Ce dernier concerne Louis Delattre et commence ainsi : « Notre généralissime est mort! » (1919d, p. 1), référence à Verhaeren. Néanmoins, malgré l'absence formelle du terme, tous les portraits de Jean Sans Peur (le pseudonyme de l'auteur des premiers portraits) sont fondés sur le champ sémantique du héros, et de nombreuses isotopies, comme celles du combat ou de l'acte de bravoure, se trouvent dans ces textes et permettent de généraliser la notion de « héros » à toutes les personnes décrites.

Nous allons étudier la rhétorique de ces portraits, afin d'analyser comment fonctionne l'utilisation du champ notionnel du "héros » dans ces biographies de littérateurs. Cette analyse vise à mettre en évidence le transfert de légitimité qui s'opère entre les faits d'arme 
B.-O. Dozo, «Le héros dans La Bataille littéraire après la Grande Guere: une représentation au service d'une littérature nationalebelge», dans Y.HAMELetM.BOUCHARD(dir),Portraitdel'hommedelettres en héros, @nalyses,hiver2006

pendant la guerre et la position occupée dans le champ littéraire, voire simplement le statut d'écrivain.

Le terme «héros", s'il est rarement utilisé pour qualifier les littérateurs, est en revanche très présent pour désigner les combattants de la guerre. Et toute la rhétorique de Jean Sans Peur sera mobilisée pour faire glisser la reconnaissance que le peuple porte à ses héros de guerre vers des écrivains qui, dans les premières biographies, sont présentés comme héroïques d'abord par leurs faits d'armes réels, puis, au fil des textes et de l'éloignement temporel de la fin de la guerre, sur un autre champ de bataille : la littérature.

L'échange dialectique entre les deux domaines est constant tout au long des portraits. Il faut examiner les modalités de contamination entre les deux pour comprendre les enjeux et la conception de la littérature qui s'y joue. Pour illustrer cette dialectique, nous allons étudier les deux premiers portraits.

Dès le premier, celui de Louis Delattre, on pourrait croire que le contrat de lecture des portraits est clair, vu que Jean Sans Peur semble honorer des écrivains qui se sont illustrés pendant la guerre.

Notre généralissime est mort; nous avons arraché leurs épaulettes à plusieurs de nos généraux qui ont tremblé devant l'ennemi; approchez, capitaine Delattre! Les vieux cadres sont à refaire, et nous voulons voir s'il n'y a pas lieu de vous appeler, avec plusieurs de vos collègues, à de plus hautes destinées. (1919d, p. 1)

Il faut trouver de nouveaux écrivains belges à honorer, car ceux qui étaient reconnus soit sont morts (comme Verhaeren, sur lequel nous reviendrons), soit ont failli à leur devoir. Une hésitation subsiste pourtant : «l'ennemi » dont il s'agit, est-ce l'ennemi allemand, ou estce une métaphore pour désigner les difficultés de la création littéraire?

Le texte se poursuit sur le mode du tribunal martial, avec passage en revue des «états de service». Or si la notion d'ennemi pouvait être douteuse (s'agissait-il de l'ennemi sur le champ militaire ou dans le 
B.-O. Dozo, «Le héros dans La Bataille littéraire après la Grande Guere: une représentation au service d'une littérature nationalebelge», dans Y.HAMELetM.BOucHARD(dir),Portraitdel'hommedelettresenhéros, @nalyses, hiver2006

champ littéraire?), les « états de service » sont fondés sur la production littéraire. Chaque œuvre produite est rapidement commentée et évaluée :

Et que nous montrez-vous là? Ah! C'est votre premier livre, Croquis d'Écolier, paru à Mons en 1888. Le drôle de petit bouquin! Comme il est solennel dans son cartonnage rouge, comme il est divertissant avec ses notations émues et déjà jolies. Mais voici mieux : vos Contes de mon Village tout chauds de votre fécond amour de la terre wallonne [...]. (1919d, p. 1)

Et ainsi de suite. On pourrait donc penser, en lisant ce premier portrait, que la «bataille littéraire » n'est qu'une métaphore de la condition de l'écrivain. Mais ce n'est pas le cas.

La deuxième biographie vient contredire cette première analyse : elle dresse le portrait de Willy Coppens, qui, assez étonnamment, ne publiera en volume que bien après la Première Guerre (en 1927, Fevilles volantes, Bruxelles, Goosens). Willy Coppens, à l'époque, est aviateur. Il a participé à de nombreuses batailles aériennes durant la guerre, où il laissera une jambe. Néanmoins, il trouve sa place dans un journal dédié à la littérature, et dont l'objectif est, d'après le premier numéro, d'« ouvrir une bataille nouvelle : c'est pour qu'elle pût avoir lieu avec toutes celles des esprits, que nos soldats ont fait l'autre, celle des corps » (1919a, p. 1).

On constate donc que les héros mis en avant sont avant tout des héros de la guerre, et qu'ils ne sont littérateurs que secondairement. Coppens est un de ceux pour qui le lien avec la littérature à l'époque est le plus ténu. Il en devient quasiment métaphorique, et se limite finalement à une comparaison entre les deux exploits :

Il s'en prenait surtout aux ballons-captifs, aux «Drachen» hideux qui balançaient dans les nues leurs corps engraissés de Dragons déchus. Ainsi, à chacun de ses coups de lance, ce chevalier des airs débarrassait l'armée d'un espion et le ciel d'une laideur... 
B.-O. Dozo, «Le héros dans La Bataille littéraire après la Grande Guere: une représentation au service d'une littérature nationalebelge», dans Y.HAMELetM.BOucHARD(dir),Portraitdel'hommedelettresenhéros, @nalyses, hiver2006

Car ne fut-il pas un prodigieux artiste à sa manière? Comme le poète il a quitté les petitesses de la terre, il a vécu de mouvements et de rythme, il s'est enivré aux vins d'or des matins; il a vu l'autre côté du nuage! (1919e, p. 1)

En 1919, la proximité de Coppens avec la littérature est toute rhétorique...

L'analyse ne laisse plus de doute, et les preuves se multiplient au long $\mathrm{du}$ dépouillement du corpus: le critère de la bravoure guerrière l'emporte sur le critère littéraire. Même Maeterlinck est évalué à cette aune. Bien sûr, la fin de l'article lui accorde d'être « à l'heure actuelle la gloire la plus haute des lettres belges » (1919k, p. 1), mais Jean Sans Peur émet quelques réserves discrètes sur l'auteur :

Le contemplatif de Sainte-Wandrille est un distillateur d'essences subtiles et parfois un peu alambiquées. [...] Cette conception de l'art durera ce qu'elle durera« : on lui peut préférer la toute simple beauté de la vie telle qu'elle est, sans les sept Princesses, les clés d'or et les portes d'ébène; mais on ne peut contester que les conceptions de Maeterlinck ne portent l'empreinte d'un art extrêmement élevé. (1919k, p. 1)

Verhaeren, au contraire, est exalté du fait de sa mort pour la patrie qui, plus encore que sa vie, lui confère un statut héroïque.

Devant ton image tourmentée, ce n'est pas tant ta gloire ni ton génie qui s'imposent d'abord à mon esprit; mais le sens plutôt de ta mort après celui que tu nous donnas de ta vie. (19191, p. 1)

Maintenant que le critère de l'hérö̈sme mis en avant par ce journal littéraire est connu, on peut dresser un inventaire des traits caractéristiques de ce héros. On retrouve ces traits au fil des articles, chaque écrivain ou homme de lettres mis en avant incarnant plus ou moins l'un ou l'autre de ces traits.

On peut dégager trois grands traits définitoires du héros vu par Jean Sans Peur: il a réalisé des actes de bravoure, qu'ils soient militaires, 
B.-O. Dozo, «Le héros dans La Bataille littéraire après la Grande Guere: une représentation au service d'une littérature nationalebelge», dans Y.HAMELetM.BoucHARD(dir),Portraitdel'hommedelettresenhéros, @nalyses, hiver2006

littéraires ou autres; il est proche du peuple, tant dans ses actes que dans ses écrits; enfin, c'est un défenseur de la nation belge, lorsqu'il est à l'étranger (en particulier à Paris) ou lorsqu'il la met en scène dans ses livres.

\section{Actes de bravoure}

On l'a vu, les actes de bravoure militaire sont les plus valorisés; une position importante durant la guerre en fait directement un écrivain intéressant : ainsi en est-il de Maurice Des Ombiaux.

L'enlumineur savoureux de l'Histoire de Saint Doden et de tant d'autres contes mirifiques connut en ces dernières années un destin inattendu et brillant. En 1915, il fut appelé au Hâvre par M. de Broqueville, qui l'attacha à son cabinet de ministre de la guerre et de président du conseil. Son premier soin fut de faire connaitre la Résistance de la Belgique envabie, dans un livre nerveux et haletant, dont les journaux des deux continents reproduisirent des extraits. (1919j, p. 1)

Les œuvres qu'il doit à cette expérience de guerre sont mises en avant, et on en souligne la diffusion importante. Le reste de sa production est à peine évoqué : c'est bien pour son rôle d'attaché au cabinet du ministre de la Guerre qu'il bénéficie d'un portrait de Jean Sans Peur. Mais les actes de bravoure militaire ne sont pas les seuls à être rapportés : Destrée est présenté comme un aventurier moderne, qui a survécu à différentes grandes expéditions, l'une dans le lointain orient, l'autre dans la Russie bolchévique. L'homme fascine par ses multiples activités. Sous la forme d'une interview, le portrait est épique, les adjectifs grandiloquents. Le tout se conclut par une dernière question :

Ce sera une délectation de lire tout cela dans vos livres... Vous en avez publié beaucoup? — Des brassées... Demandez à Dupierreux, il vous mettra au courant. Je vous quitte, Jean Sans Peur, car je suis pressé... (1919h, p. 1) 
B.-O. Dozo, «Le héros dans La Bataille littéraire après la Grande Guere: une représentation au service d'une littérature nationale belge», dans Y.HAMELetM.BOucHARD(dir),Pontraitdel'hommedelettresenhéros, @nalyses, hiver2006

Cette façon de mettre par écrit cette fausse interview donne une impression de «starification» de Destrée l'aventurier. Il n'est pas question de ses ouvrages, sauf pour souligner leur quantité (mais pas leur qualité). Le peu de cas fait de ceux-ci indique combien la personne et les actions de Destrée importent plus que ses œuvres littéraires, reléguées au rang de comptes rendus des agissements de l'homme.

Les portraits de La Bataille littéraire, on l'a vu, ne concernent pas tous la littérature : il y est question du docteur Depage. C'est son action en tant que médecin qui lui vaut une biographie dans ces pages. La guerre grandit encore cette action, au point que Jean Sans Peur y insiste :

La guerre est venue humaniser encore, et mêler à la vie quotidienne, ceux-là qui, tout en se tenant au-dessus d'elle, par l'élévation de leurs soucis, ne vivaient que pour la foule, pour l'instruire, lui apprendre la beauté et la joie, la guérir de ses maux, l'aimer. (1919i, p. 1)

Cette citation me permet de passer au deuxième trait définitoire du héros selon La Bataille littéraire: sa proximité avec le peuple.

\section{La proximité avec le peuple}

Cette qualité est rarement soulignée quand celui dont le portrait est fait la possède; en revanche, lorsqu'il en est dépourvu, Jean Sans Peur ne manque pas de la rappeler. Dès l'éditorial du premier numéro, les rédacteurs de La Bataille littéraire insistent sur l'implication sociale qu'ils veulent donner à la littérature.

Finies les aristocraties des petits cénacles, à bas les tours d'ivoire, les Parnasses inaccessibles, et, aussi, les smokings des aèdes de salon! Nous ne sommes pas des «hors-du-siècle»: nous voulons nous mêler aux hommes et aux choses de chaque jour, aux efforts des foules que nous écouterons vivre, c'est-àdire souffrir et espérer. (1919a, p. 1) 
B.-O. Dozo, «Le héros dans La Bataille littéraire après la Grande Guere: une représentation au service d'une littérature nationalebelge», dans Y.HAMELetM.BOUCHARD(dir),Portraitdel'hommedelettres en héros, @nalyses,hiver2006

Ces «hors-du-siècle » conspués réfèrent en fait à une œuvre homonyme d'Albert Giraud. Ce dernier est un des écrivains écoutés à l'époque (on le trouve à la fondation de l'Académie royale de langue et littérature française un an plus tard), mais ses prises de position en faveur de l'Art pour l'Art ne semblent permettre que difficilement à La Bataille littéraire de l'intégrer à son panthéon d'auteurs. Pourtant, dès le troisième numéro, un portrait lui est consacré. Il faut observer la rhétorique mise en place par Jean Sans Peur pour évoquer l'écrivain :

À l'heure où nous cherchons des maîtres, Albert Giraud se dresse devant nous, dans une solitude que ses vers ont tant de fois souhaitée. L'élan de notre art et de nos consciences aurait déjà dû s'élever vers lui; et si nous n'avons pas mis ce journal des écrivains belges sous l'égide — et le masque — de ce somptueux poète, c'est à cause d'un scrupule - et d'un trouble. (1919f, p. 1)

On apprend plus loin que Giraud, contrairement à d'autres, n'a pas ployé «sous le joug» de l'ennemi et que, "malgré qu'il eût tant médit d'elle, [il] a su comprendre l'âme d'un peuple et lui montrer la dignité » (1919f, p. 1). Sauvé par son comportement durant la guerre, Giraud est présenté dans La Bataille littéraire parce que Jean Sans Peur lui prête après la guerre une prise de conscience des sacrifices du peuple :

L'héroïsme, la volonté, l'endurance d'un monde qu'on croyait veule en face d'un autre qui s'affirmait tout-puissant, le sacrifice anonyme de millions d'êtres frustes que les poètes croient pouvoir mépriser, ah! leçon de charité humaine, de simplicité qui peut se passer des vers - et des mots! (1919f, p. 1)

Le même type de reproche - un certain mépris du peuple - est adressé à Maeterlinck, et dans une moindre mesure à Verhaeren. Mais Jean Sans Peur les réhabilite tous deux au moyen de stratégies rhétoriques similaires.

Enfin, cette proximité avec le peuple est mise en avant lorsque l'auteur dépeint dans ses œuvres la vie populaire. George Garnir est de ceux-là, 
B.-O. Dozo, «Le héros dans La Bataille littéraire après la Grande Guere: une représentation au service d'une littérature nationalebelge», dans Y.HAMELetM.BOUCHARD(dir),Portraitdel'hommedelettres en héros, @nalyses,hiver2006

lui qui a, d'après Alix Pasquier qui signe certains des portraits, «consacré aux sites et aux paysans du Condroz des pages que [Pasquier] ne [s]e lassera jamais de relire » (1919b, p. 1)

Cette mise en avant du régionalisme permet de faire la transition vers le troisième et dernier trait définitoire des qualités du héros vu par La Bataille littéraire: les défense et illustration de la nation belge.

\section{Défense et illustration de la Belgique}

Ce dernier point est le plus présent : au sortir de la guerre, comme nous l'avons rappelé dans l'introduction, la Belgique n'est plus un petit pays (la formulation est importante : on la retrouve telle quelle — « la Belgique a cessé d'être un petit pays » — dans le premier éditorial de la revue). Or, pour défendre cette idée, il faut d'un côté des gens pour l'illustrer, quitte à puiser dans un passé prestigieux (les peintres flamands de la Renaissance), et de l'autre des gens pour la promouvoir.

Sont ainsi mises en avant des personnalités comme Henri Pirenne, historien belge défenseur de la thèse d'une unité de la Belgique qui remonterait bien avant sa constitution en État en 1830 (elle remonterait en fait au traité de Verdun en 843). Jean Sans Peur le souligne :

$\mathrm{Au}$ point de vue belge, Pirenne a droit à l'immense reconnaissance de ses compatriotes. Il a joué un rôle primordial et peut-être unique dans la formation de la conscience nationale. (1919g, p. 1)

D'autres encore sont cités à ce titre, par exemple Henri Carton de Wiart pour ses romans historiques, Hippolyte Fierens-Gevaert pour ses ouvrages sur les peintres flamands, ou encore Franz Hellens pour son style si particulier, qu'il qualifiera lui-même de «réalisme magique » et qui est en quelque sorte lié à une «identité » littéraire belge. 
B.-O. Dozo, «Le héros dans La Bataille littéraire après la Grande Guere: une représentation au service d'une littérature nationalebelge», dans Y.HAMELetM.BOUCHARD(dir),Portraitdel'hommedelettres en héros, @nalyses,hiver2006

La capacité de l'auteur à se faire connaître et reconnaitre en dehors de la Belgique, et particulièrement en France, constitue aussi un élément de l'illustration de la Belgique. Dès qu'un auteur belge a un lien quelconque avec l'étranger, Jean Sans Peur ne manque pas de le signaler. On peut citer Maurice Des Ombiaux, ou Horace Van Offel, dont la collaboration avec l'occupant durant la Seconde Guerre mondiale donne un écho étonnant à sa présence dans ce panthéon. Jean Sans Peur écrit fièrement de lui :

Depuis 3 ans Van Offel se réalise à Paris, dans le firmament duquel nous sommes heureux de voir son étoile monter et resplendir. [...] À un des nôtres qui lutte à Paris que pouvonsnous dire ici comme paroles qui l'encouragent et le forcent à vaincre? C'est que les jeunes écrivains de Belgique ont les yeux tournés vers lui et qu'ils distinguent parfaitement les Élus de la gloire, — des médisants et des imbéciles. (1919m, p. 1)

En mettant ainsi en évidence le lieu de consécration littéraire de l'époque (Paris), et en insistant sur la reconnaissance qu'il est nécessaire d'acquérir là-bas même pour un auteur belge, La Bataille littéraire rend visible la tension forte qui traverse le champ littéraire belge de l'époque. Malgré la nouvelle grandeur du pays, sur le marché des biens symboliques, c'est toujours Paris qui contrôle les valeurs. La revue a beau s'inscrire depuis sa première parution dans une démarche patriotique, glorifiant ses héros militaires et littéraires, au fil des numéros, les regards se tournent de plus en plus vers le centre français.

\section{Le héros littéraire belge, sa langue et la France}

Ainsi, dans le même numéro que le portrait de Van Offel, La Bataille littéraire remercie les quelques journaux français qui ont signalé son existence, et souligne la proximité d'esprit et de cœur avec la France.

Mais que signifie cette proximité? À une époque où les conflits linguistiques sont de plus en plus francs, où les revendications flamandes vont reprendre de plus belle après la parenthèse de la 
B.-O. Dozo, «Le héros dans La Bataille littéraire après la Grande Guere: une représentation au service d'une littérature nationalebelge», dans Y.HAMELetM.BOUCHARD(dir),Portraitdel'hommedelettres en héros, @nalyses,hiver2006

guerre, où le suffrage universel (mais uniquement pour les hommes) met en évidence le clivage linguistique qui traverse le pays, la position unitariste de La Bataille littéraire, défendue par une rhétorique fondée sur l'héroïsme national, peut être considérée comme une position d'arrière-garde. Et la bouée de sauvetage tardive que constitue la proximité de cœur et d'esprit postulée par rapport à la France n’existe que pour souligner le naufrage d'une conception de la Belgique dépassée déjà à l'époque.

Ainsi, à partir du numéro 35, les portraits changent d'auteur: Jean Sans Peur laisse progressivement la place à différentes personnes nommément identifiables (tels Alix Pasquier ou G.-M. Rodrigue). Cela induit pour la revue un repositionnement éditorial stratégique par rapport à la question identitaire : les marques de l'unité belge vont progressivement disparaittre, suppléées par un rapprochement avec la France.

Le numéro 44 entérine ce revirement: le lieu d'édition apparaît en grand au-dessous du titre (Paris - Bruxelles), alors qu'il se faisait très discret lorsque l'adresse ne mentionnait que Bruxelles, et la première page est barrée d'un titre proclamant: «À la France ». Ce titre est répété en sous-titre d'un poème et d'un éditorial de part et d'autre de la page, et Georges Clemenceau est la figure centrale dont on brosse le portrait. L'éditorial commence par «France, c'est vers toi que monte le premier cri d'allégresse de notre Belgique délivrée » (1919c, p. 1), et contient la phrase: «c'est ta littérature qui est la première littérature» (1919c, p. 1). L’entreprise mise sur pied depuis le premier numéro est recadrée, comme le laissait percevoir différentes évolutions dans la revue: la littérature belge et son panthéon sont dans une relation de dépendance forte par rapport à la France.

La conception du héros littéraire belge défendue par la revue est une conception à usage interne à la Belgique, qui lui a permis de prendre position par rapport aux volontés séparatistes d'une certaine frange de la population belge. Le héros est le héraut d'une nation unie, qui a fait face à l'ennemi. Ses qualités sont plus humaines qu'artistiques. On retient surtout de lui ses exploits sur le champ de bataille en tant que 
B.-O. Dozo, «Le héros dans La Bataille littéraire après la Grande Guere: une représentation au service d'une littérature nationalebelge», dans Y.HAMELet M.BOUCHARD(dir),Portraitdel'hommedelettres en héros, @nalyses, hiver2006

défenseur de la Belgique unie. Mais dès qu'il s'agit de littérature, c'est à Paris que la bataille littéraire a lieu.

\section{Bibliographie}

[ANONYME]. 1919a, "Premiers coups de canon », La Bataille littéraire, $\mathrm{n}^{\circ} 1,23$ janvier.

Aron, Paul et Pierre-Yves Soucy. 1998, Les revues littéraires belges de langue française de 1830 à nos jours, Bruxelles, Labor, [1993] édition revue et augmentée, coll. « Archives du futur».

Destrée, Jules. 1922, "Création de l'Académie. Rapport au Roi », Bulletin de l'Académie royale de Langue et Littérature françaises, t. I.

PASQUIER, Alix. 1919b, "Silhouette. George Garnir", La Bataille littéraire, $\mathrm{n}^{\circ}$ 35, 25 septembre.

-. 1919c, « À la France », La Bataille littéraire, n 44, 4 décembre.

SANS PeUR, Jean. 1919d, "Silhouette. Louis Delattre», La Bataille littéraire, $\mathrm{n}^{\circ} 1,23$ janvier.

—. 1919e, «Silhouette. Willy Coppens », La Bataille littéraire, 30 janvier, $\mathrm{n}^{\mathrm{o}} 2$.

—. 1919f, "Silhouette. Albert Giraud», La Bataille littéraire, $\mathrm{n}^{\circ} 3$, 6 février.

—. 1919g, "Silhouette. Henri Pirenne», La Bataille littéraire, $\mathrm{n}^{\circ} 3$, 6 février.

—. 1919h, «Silhouette. Jules Destrée », La Bataille littéraire, n 4, 13 février.

—. 1919i, «Silhouette. Le Docteur Depage », La Bataille littéraire, n 6, 27 février. 
B.-O. Dozo, «Le héros dans La Bataille littéraire après la Grande Guere: une représentation au service d'une littérature nationalebelge», dans Y.HAMELetM.BOUCHARD(dir),Portraitdel'hommedelettres en héros, @nalyses,hiver2006

—. 1919j, "Silhouette. Maurice Des Ombiaux », La Bataille littéraire, $\mathrm{n}^{\circ} 7,6$ mars.

—. 1919k, «Silhouette. Maurice Maeterlinck», La Bataille littéraire, $\mathrm{n}^{\mathrm{o}} 19,1^{\mathrm{er}}$ juin.

—. 19191, «Silhouette. Émile Verhaeren », La Bataille littéraire, n 20, 8 juin.

—. 1919m, «Silhouette. Horace Van Offel », La Bataille littéraire, n 33, 11 septembre. 